\title{
Erfolgreiche Etablierung der MRT als Therapiemarker für neuroinfektiologische Erkrankungen
}

Spätestens seit dem Ausbruch der SARSCoV-2-Pandemie rücken infektiologische und neuroinfektiologische Erkrankungen wieder zunehmend in den Fokus der Medizin und der Öffentlichkeit. Bei der Diagnose und Behandlung solcher Erkrankungen, etwa der progressiven Multifokalen Leukenzephalopathie, kann die Magnetresonanztomografie eine wichtige Rolle einnehmen. Das zeigt ein internationales Forscherteam um den Neuroradiologen Prof. Dr. Dr. Mike P. Wattjes von der Medizinischen Hochschule Hannover in einer aktuellen Publikation.

Bei der Diagnose bestimmter neuroinfektiologischer Erkrankungen ist die Magnetresonanztomografie (MRT) des Gehirns, aber auch des Rückenmarks ein fester Bestandteil der klinischen Diagnostik. Die MRT wird besonders häufig zur Diagnose der progressiven Multifokalen Leukenzephalopathie (PML) eingesetzt, einer Infektion des Gehirns, die durch das JC-Virus ausgelöst wird. Besonders von Infektionen mit diesem Virus können Menschen betroffen sein, die an HIV und anderen systemischen immunsuppressiven Erkrankungen leiden, aber auch Patientinnen und Patienten mit rheumatischen Erkrankungen oder Multipler Sklerose, die mithilfe immunsuppressiver Therapien behandelt werden.

Da es für die PML lange keine Therapien gab, beschränkte sich die Rolle der MRT-
Bildgebung nahezu ausschließlich auf die Diagnose und die Dokumentation des Krankheitsverlaufs. Aber mit der Einführung neuer Therapiestrategien, wie etwa der Stimulierung der zellulären Immunantwort auf die Erkrankung durch sogenannte Checkpoint-Inhibitoren und die Gabe virusspezifischer allogener T-Zellen, sind zuverlässige Marker zur Erfassung des TherapieAnsprechens und des Monitorings der PML im Allgemeinen notwendig geworden. Auf Initiative der Amerikanische Behörde für Lebens- und Arzneimittel (U.S. Food and Drug Administration, FDA) und der amerikanischen Gesundheitsbehörde (National Institutes of Health, NIH) werden deshalb entsprechende Marker identifiziert und untersucht.

Prof. Dr. Dr. Mike P. Wattjes, Leitender Oberarzt am Institut für Neuroradiologie der Medizinischen Hochschule Hannover (MHH), hat mit Kolleginnen und Kollegen aus der FDA und dem NIH sowie weiteren internationalen Expertinnen und Experten auf diesem Gebiet die MRT als vielversprechenden Marker für das Therapie-Monitoring bei der PML ausgemacht. Die Forschungsergebnisse von Professor Wattjes und seinen Kolleginnen und Kollegen sind vor Kurzem in der renommierten Fachzeitschrift Brain veröffentlicht worden (Baldassari LE \& Wattjes MP, et al. The neuroradiology of progressive multifocal leukoencephalopathy: a clinical trial perspective. Brain 2021 Nov 16; awab419. doi: 10.1093/brain/awab41.).

„Die MRT des Gehirns ist eine sensitive und sichere Methode, nicht nur, um die Diagnose einer PML frühzeitig zu stellen, sondern auch, um die Krankheitsprogression und das Ansprechen auf die Therapie sehr gut zu dokumentieren und zu beobachten“, erläutert Professor Wattjes. „Neuere Studien unter anderem in interdisziplinärer Zusammenarbeit mit der Neurologischen Klinik der MHH haben gezeigt, dass unter erfolgreicher T-Zell-Therapie Veränderungen der PML nicht weiter fortschreiten und sich zum Teil sogar zurückbilden können.“ Laut Professor Wattjes gebe es Hinweise darauf, dass das durch die MRT dokumentierte Ansprechen auf die Therapie auch mit anderen Markern wie beispielsweise der Viruslast im Liquor in Zusammenhang stehe. Welche mikrostrukturellen Veränderungen für den Rückgang der Veränderungen verantwortlich sind, wird gegenwärtig weiter untersucht. „Das Beispiel der erfolgreichen Etablierung der Bildgebung als Therapiemarker der PML sollte uns ein Ansporn sein, dies auch für andere neuro-infektiologische Erkrankungen zu untersuchen und zu etablieren. Die nächsten neuroinfektiologischen Herausforderungen mit hoffentlich neuen therapeutischen Möglichkeiten kommen bestimmt“, sagt Professor Wattjes. 